\title{
Traumatic Brain Stem Injury: Evaluation by MRI
}

read with great interest the article by Hilario et al. ${ }^{1}$ The authors retrospectively analyzed the MR imaging data from the first 30 days after injury in 108 patients with severe head trauma and found that the presence of posterior and bilateral brain stem injuries were poor prognostic signs. Although they analyzed the location of the brain stem lesions, they did not analyze the volume or depth of the brain stem lesions. In their study, the median time between trauma and MR imaging was 17 days, with a maximum of 30 days. We previously investigated the MR imaging findings of traumatic primary brain stem injury. ${ }^{2}$ In our study, MR imaging was carried out within 6 days, generally 2 days after the injury. ${ }^{2}$ Superficial dorsal brain stem injury was found to be an indicator of a good prognosis, whereas only deep dorsal brain stem injury was related to a poor prognosis. Small lesions may disappear in a few days after injury. Most of the injuries in Hilario et al's series of patients had affected the posterior dorsolateral aspect of the midbrain. Hilario et al's study may have underestimated the lesion size and presence of brain stem injury. They did not extensively discuss the cause of unilateral injuries with a good prognosis, except for the possibility of supratentorial herniation, and they did not discuss the clinical and MR imaging findings of supratentorial herniation. Our study excluded secondary brain stem injury associated with cerebral herniation and evaluated only "primary" brain stem injury. We discussed 2 mechanisms of brain stem injury: primary brain stem injury that occurs after a direct impact of the brain stem against the tentorial free edge, and brain stem injury associated with diffuse axonal injury. Direct focal brain

http://dx.doi.org/10.3174/ajnr.A3555 stem injuries caused by an impact against the free tentorial edge have been pathologically and radiologically recognized. ${ }^{3,4}$ It is sometimes difficult to differentiate these 2 mechanisms of injury; however, the brain stem lesion size and the location and supratentorial lesion findings are helpful. Hilario et al's report did not discuss the mechanism of brain stem injury. I believe that differentiating these 2 mechanisms of brain stem injury is therefore critical for accurately diagnosing and understanding traumatic brain stem injuries. In Hilario et al's study, nonhemorrhagic injuries showed the highest positive predictive value for a good outcome, though no explanation was discussed. Obviously, the presence of no injuries would be most predictive of a good outcome.

\section{REFERENCES}

1. Hilario A, Ramos A, Millan JM, et al. Severe traumatic head injury: prognostic value of brain stem injuries detected at MRI. AJNR Am J Neuroradiol 2012;33:1925-31

2. Shibata Y, Matsumura A, Meguro K, et al. Differentiation of mechanism and prognosis of traumatic brain stem lesions detected by magnetic resonance imaging in the acute stage. Clin Neurol Neurosurg 2000;102:124-28

3. Clifton G, McCormick W, Grossman R. Neuropathology of early and late deaths after head injury. Neurosurgery 1981;8:309-14

4. Firsching R, Woischneck D, Diedrich M, et al. Early magnetic resonance imaging of brainstem lesions after severe head injury. J Neurosurg 1998;89:707-12

Y. Shibata

Department of Neurosurgery University of Tsukuba Mito Medical Center Ibaraki, Japan 